\title{
Dead-time optimized time-correlated photon counting instrument with synchronized, independent timing channels
}

Michael Wahl, Hans-Jürgen Rahn, Ingo Gregor, Rainer Erdmann, and Jörg Enderlein

Citation: Review of Scientific Instruments 78, 033106 (2007);

View online: https://doi.org/10.1063/1.2715948

View Table of Contents: http://aip.scitation.org/toc/rsi/78/3

Published by the American Institute of Physics

\section{Articles you may be interested in}

Scalable time-correlated photon counting system with multiple independent input channels

Review of Scientific Instruments 79, 123113 (2008); 10.1063/1.3055912

Full correlation from picoseconds to seconds by time-resolved and time-correlated single photon detection

Review of Scientific Instruments 76, 083104 (2005); 10.1063/1.1946088

Integrated multichannel photon timing instrument with very short dead time and high throughput

Review of Scientific Instruments 84, 043102 (2013); 10.1063/1.4795828

A study of pile-up in integrated time-correlated single photon counting systems

Review of Scientific Instruments 84, 103105 (2013); 10.1063/1.4824196

Inexpensive electronics and software for photon statistics and correlation spectroscopy

American Journal of Physics 82, 712 (2014); 10.1119/1.4869188

Improving the counting efficiency in time-correlated single photon counting experiments by dead-time optimization

Review of Scientific Instruments 86, 113101 (2015); 10.1063/1.4934812

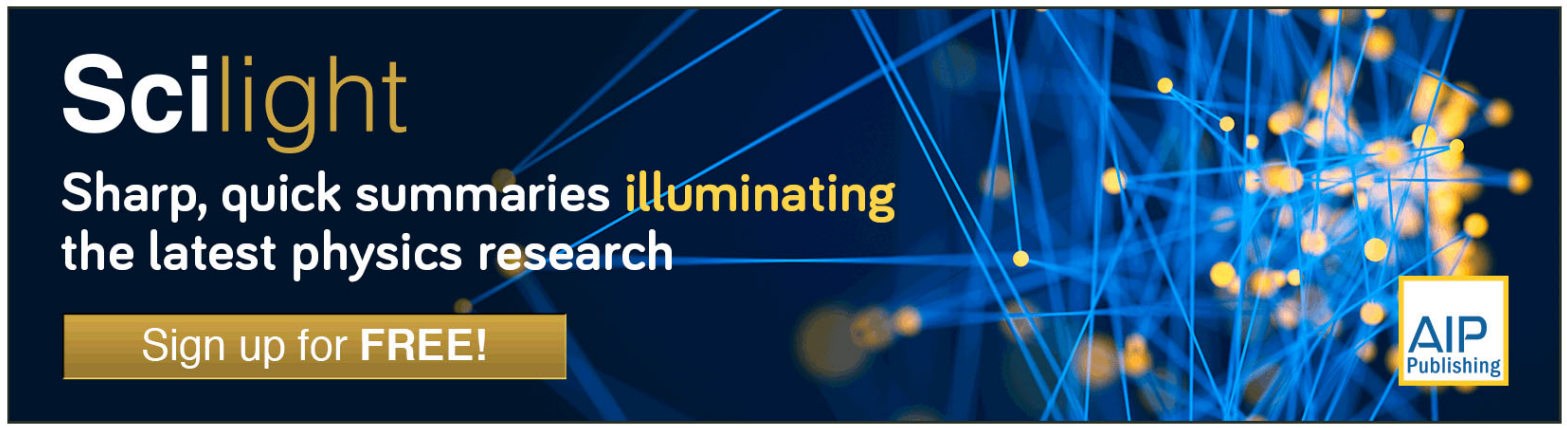




\title{
Dead-time optimized time-correlated photon counting instrument with synchronized, independent timing channels
}

\author{
Michael Wahl ${ }^{\text {a) }}$ and Hans-Jürgen Rahn \\ PicoQuant GmbH, Rudower Chaussee 29, D-12489 Berlin, Germany \\ Ingo Gregor \\ Institute for Neurobiology and Biophysics 1, Forschungszentrum Jülich, D-52425 Jülich, Germany \\ Rainer Erdmann \\ PicoQuant GmbH, Rudower Chaussee 29, D-12489 Berlin, Germany \\ Jörg Enderlein \\ Institute for Neurobiology and Biophysics 1, Forschungszentrum Jülich, D-52425 Jülich, Germany
}

(Received 15 December 2006; accepted 19 February 2007; published online 23 March 2007)

\begin{abstract}
Time-correlated single photon counting is a powerful method for sensitive time-resolved fluorescence measurements down to the single molecule level. The method is based on the precisely timed registration of single photons of a fluorescence signal. Historically, its primary goal was the determination of fluorescence lifetimes upon optical excitation by a short light pulse. This goal is still important today and therefore has a strong influence on instrument design. However, modifications and extensions of the early designs allow for the recovery of much more information from the detected photons and enable entirely new applications. Here, we present a new instrument that captures single photon events on multiple synchronized channels with picosecond resolution and over virtually unlimited time spans. This is achieved by means of crystal-locked time digitizers with high resolution and very short dead time. Subsequent event processing in programmable logic permits classical histogramming as well as time tagging of individual photons and their streaming to the host computer. Through the latter, any algorithms and methods for the analysis of fluorescence dynamics can be implemented either in real time or offline. Instrument test results from single molecule applications will be presented. (C) 2007 American Institute of Physics.
\end{abstract}

[DOI: $10.1063 / 1.2715948]$

\section{INTRODUCTION}

In a classical time-correlated single photon counting (TCSPC) experiment, a sample containing luminescent entities (e.g., fluorescent dye molecules, quantum dots, or beads) is periodically excited by light pulses. The duration of the excitation has to be much shorter than the lifetime of the emission process, and excitation intensity is tuned to guarantee single photon resolved light detection by a suitable detector. For each detection event, the TCSPC electronics measures the time lag between excitation pulse and emission photon. ${ }^{1}$ Figures of merit for TCSPC electronics are the precision of the time measurement and the duration of the data processing resulting in the dead time of the electronics, during which processing of further detection events is impossible.

In early applications of TCSPC the only objective was the collection of decay curves and the instrument response function (IRF) for calibration or decay deconvolution. Therefore, immediate hardware-based histogramming of the startstop times was a natural choice for efficient data handling. In that case, one uses only the time between excitation pulse and subsequent photon emission.

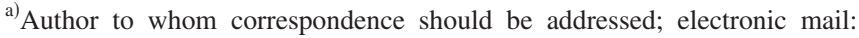
wahl@pq.fta-berlin.de
}

It was soon realized that other aspects of the photon arrival times were of equally great value in the context of single molecule fluorescence detection and spectroscopy. For instance, in single molecule experiments in flow capillaries, an important option is to identify the molecules passing through the detection volume based on their fluorescence lifetime. Each molecule transit is detected as a burst of fluorescence photons. Each time such a transit is detected its fluorescence decay time has to be determined. ${ }^{2}$ Also in the area of single molecule detection and spectroscopy, photon coincidence correlation techniques were adopted to observe antibunching effects that can be used to determine the number of observed emitters as well as the fluorescence lifetime.

Another important method that makes use of temporal photon density fluctuations over a wider time range is fluorescence correlation spectroscopy (FCS). From the fluorescence intensity fluctuations of molecules diffusing through a confocal volume, one can obtain information about the diffusion constant and the number of molecules in the observed volume. ${ }^{3}$ This allows sensitive fluorescence assays based on molecule mobility and colocalization, applicable under native conditions and on minute sample volumes. Due to the small numbers of molecules, the photon count rates in FCS are fairly small. Therefore the only practical way of collect- 
ing the data is by means of single photon counting. In order to obtain the time resolution of interest for the diffusion processes, counting with microsecond resolution is required. Hardware correlators for FCS can be implemented very efficiently and recent designs are widely used. ${ }^{4}$ However, these instruments are dedicated to correlation with nanosecond resolution at best and cannot perform picosecond TCSPC.

The requirements of these analytical techniques based on single photon count data have much in common. Indeed, all of them can be implemented with the same experimental setup and are based on photon arrival times. A first step towards unified instrumentation for this purpose was a modification of classical TCSPC electronics. The start-stop timing circuitry is used as previously, providing the required picosecond resolution for TCSPC. In order to maintain the information embedded in the temporal patterns of photon arrivals the events are stored as separate records. In addition to that, a coarser timing (time tagging) is performed on each photon event with respect to the start of the experiment. This is called the time-tagged time-resolved (TTTR) data collection. ${ }^{5}$ In this scenario the different time scales are processed and used rather independently. However, it is of great interest to obtain high resolution timing on the overall scale, i.e., combining coarse and fine timings into one global arrival time figure per event, with picosecond resolution. In a most generic approach, without implicit assumptions on start and stop events, one would ideally just collect precise time stamps of all events of interest (excitation, emission, or others) with the highest possible throughput and temporal resolution, and then perform the desired analysis on the original event times. Ideally this would be done on independent channels, so that between channels even dead-time effects can be eliminated. Here we present such an instrument. It enables temporal analysis from picosecond to second time scale, thereby covering almost all dynamic effects of the photophysics of fluorescing molecules. At the same time it can be used for all conventional histogramming applications.

\section{INSTRUMENT}

\section{A. TDC based TCSPC electronics}

The actual time difference measurement in TCSPC is done by means of fast electronics which provide a digital timing result. Depending on requirements such as time resolution and throughput there are several solutions for this. Slow (i.e., microsecond) fluorescence or luminescence processes may be recorded with resolutions of a few nanoseconds or more. In such cases digital counters are very convenient for the timing and, if designed well, may even be free of dead time. However, substantially higher time resolution demands exist in most fluorescence lifetime measurements with organic dyes of practical importance in biochemical fluorescent labeling. Here the required resolutions are on the order of a few picoseconds. The historically most common timing solution is the combination of time to amplitude converters (TACs) and analog to digital converters (ADCs) allowing time bin widths down to less than a picosecond. TAC and ADC are essentially a stopwatch suitable for short term measurements at high resolution but not suited to obtain tim-

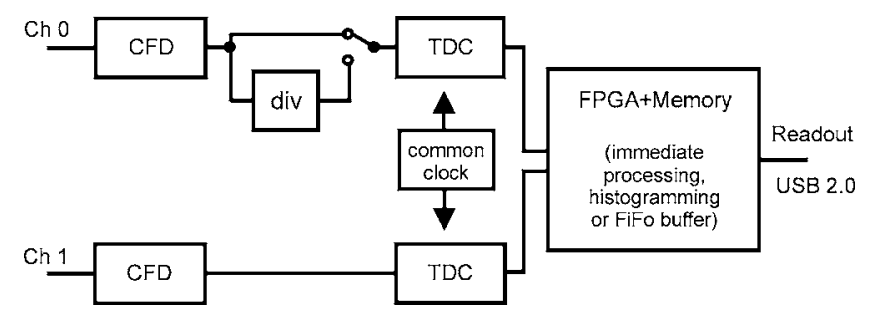

FIG. 1. Block diagram of the instrument. The control paths are not shown.

ing information on multiple events across the whole experiment of many excitation/emission cycles. This is a limitation for advanced analysis of fluorescence dynamics or other applications in quantum physics. However, the timing today can be carried out by a dedicated timing circuit, a so-called time to digital converter (TDC). A review on the various implementations is given by Kalisz. ${ }^{6}$ Recently TDCs are approaching a resolution of $1 \mathrm{ps}$, while providing a crystallocked calibration. Most TDCs can operate repeatedly and continuously on multiple events. They also can measure virtually unlimited time differences by means of digital counters (coarse scale) and interpolation on a picosecond scale. In order to keep the picosecond interpolator permanently calibrated it is usually tied to the coarse clock crystal oscillator through a control loop. This concept permits exceptionally small, compact, and affordable TCSPC solutions, as the circuits can be implemented as hybrid chips or application specific integrated circuits (ASICs) at relatively low cost, low power consumption, and high reliability.

\section{B. Specific instrument design}

The chosen TDC design offers a resolution of $4 \mathrm{ps}$ and a dead time of $90 \mathrm{~ns}$. As opposed to the conventional start-stop measurement, the instrument has two independent time digitizers. In the instrument's histogramming mode, they can be used for start-stop measurements as usual, while the startstop time differences are calculated in a programmable hardware and then passed to the histogrammer or other processing. Figure 1 shows a block diagram of the instrument. Both inputs have a constant fraction discriminator (CFD) in order to handle fluctuating pulse heights. A divider in channel 0 can be used if high sync frequencies are present. Otherwise it is bypassed (see also sec. II C). The two TDCs are locked to the same crystal clock, so that their time measurements are always synchronized and equally calibrated. The event timings may be regarded as precise (picosecond) wall clock readings. Therefore, the subtraction or comparison of event times is valid within and across the channels. Due to the TDC based time measurement, the time difference between events (e.g., start and stop) can be arbitrarily long, while still being determined at the full resolution. Relative error is determined only by the crystal characteristics. Only due to practical limits of the histogram storage the histogramming mode is limited to 65535 time bins. The bin width can also be increased (binary multiples of the 4 ps base resolution) in order to increase the overall time span (here maximum of $32 \mu \mathrm{s})$. Since the dead time is acting only within each channel, at slow excitation rates the histogrammer can process multiple photons per excitation/emission cycle. Hence, it can 
collect data much more efficiently than a TAC based system which always needs to wait for the next stop (sync) event. Another interesting feature resulting from this approach is that the histogram can be collected at the full resolution, but with an arbitrary offset. This is similar to employing a bias in TAC based systems but allows much larger shifts (here maximum of $100 \mu \mathrm{s})$. This is of particular interest in ranging and time of flight applications.

The system is to a large extent implemented in a field programmable gate array (FPGA). In addition to conventional histogramming, the FPGA design provides two time tagging measurement modes that allow for capturing event arrival timing with picosecond resolution on a "global" laboratory time scale. As outlined earlier, the concept of two independent TDCs is especially powerful in continuous data collection of individual events for more detailed analysis beyond TCSPC histogramming.

\section{Time tagging data acquisition modes 1. $T 2$ mode}

The difference of the two time tagging modes is primarily in the handling of sync events from, e.g., a pulsed laser. In $\mathrm{T} 2$ mode both signal inputs are functionally identical. Usually both inputs are used to connect photon detectors. The divider is bypassed. The events from both channels are recorded independently and treated equally. In each case an event record is generated that contains information about the channel it came from and the arrival time of the event with respect to the overall measurement start. The timing is recorded with 4 ps resolution. Each T2 mode event record consists of 32 bits. There are 4 bits for the channel number and 28 bits for the time tag. If the time tag overflows, a special overflow marker record is inserted in the data stream, so that upon processing of the data stream a theoretically infinite time span can be recovered at full resolution. Autocorrelations can therefore be calculated at the full resolution, but only starting from lag times larger than the dead time (90 ns typically). However, dead times, including those of the detectors, exist only within each channel but not across the two channels. Therefore, cross correlations can be calculated down to zero lag time. This allows powerful applications such as FCS with lag times from picoseconds to hours. In order to achieve this, previous implementations had to solve the problem of synchronizing and jointly calibrating two TAC based instruments as well as correcting for TAC nonlinearities and related artifacts. ${ }^{7}$

The $\mathrm{T} 2$ event records are queued in a first in first out (FIFO) buffer capable of holding up to 256000 event records. The FIFO input is fast enough to accept records at the full speed of the time digitizers (up to 10 $\times 10^{6}$ counts $/ \mathrm{s}$ each). This means even during a fast burst no events will be dropped except those lost in the dead time anyhow. The FIFO output is continuously read by the host PC, thereby making room for fresh incoming events. Even if the average read rate of the host PC is limited, bursts with much higher rate can be recorded for some time. Only if the average count rate over a longer period of time exceeds the readout speed of the PC, a FIFO overrun could occur. Then the measurement must be aborted because data integrity cannot be maintained. However, on a modern PC (2.8 GHz Pentium 4, Windows XP) sustained average count rates over $5 \times 10^{6}$ counts/s were obtained. This total transfer rate must be shared by the two input channels. For all practically relevant fluorescence detection applications the effective rate per channel is more than sufficient because single photon statistics are the limiting factor.

\section{T3 mode}

In T3 mode one input channel is dedicated to a sync signal. As far as the experimental setup is concerned, this is similar to TCSPC histogramming mode. The main objective is to allow high sync rates from mode locked lasers (up to $85 \mathrm{MHz}$ ) which could not be handled in T2 mode due to dead time. Accommodating the high sync rates in T3 mode is achieved as follows: First, the sync divider is employed (as in histogramming mode). This reduces the sync rate so that the channel dead time is no longer a problem. A divider of maximum of 8 will allow sync rates as high as $85 \mathrm{MHz}$. The sync events that have been divided out will be reconstructed in subsequent processing. The remaining problem is now that even with a divider of 8 , the sync rate is still too high for collecting all individual sync events like ordinary T2 mode events. Considering that sync events are not of primary interest, the solution is to record them only if they arrive in the context of a photon event on channel 1 . The event record is then composed of two timing figures: (1) the start-stop timing difference between the photon event and the last sync event, and (2) the arrival time of the event pair on the overall experiment time scale (the time tag). In the original TTTR mode concept ${ }^{5}$ the latter was obtained from an independent asynchronous clock. This made it difficult to combine the start-stop timing with the time tag or to know the sync period the event belonged to. Here another approach was chosen. The time tag is now obtained by counting sync pulses. From the T3 mode event records it is therefore possible to precisely determine which sync period a photon event belongs to. Since the sync period is also known precisely, this furthermore allows reconstructing the arrival time of the photon with respect to the overall experiment time.

Each T3 mode event record consists of 32 bits. There are 4 bits for the channel number, 12 bits for the start-stop time, and 16 bits for the sync counter. If the counter overflows, a special overflow marker record is inserted in the data stream, so that upon processing of the data stream a theoretically infinite time span can be recovered. The 12 bits for the start-stop time difference cover a time span of $4096 R$, where $R$ is the chosen resolution. At the highest possible resolution of 4 ps this results in a span of $16 \mathrm{~ns}$. If the time difference between the photon and the last sync event is larger, the photon event cannot be recorded. This is the same as in histogramming mode, where the number of bins is also finite. However, by choosing a suitable sync rate and a compatible resolution $R$, it is possible to reasonably accommodate all relevant experiment scenarios. $R$ can be chosen in doubling steps between 4 and 512 ps. The data transfer uses a FIFO buffer as in T2 mode. Again, sustained average count rates 


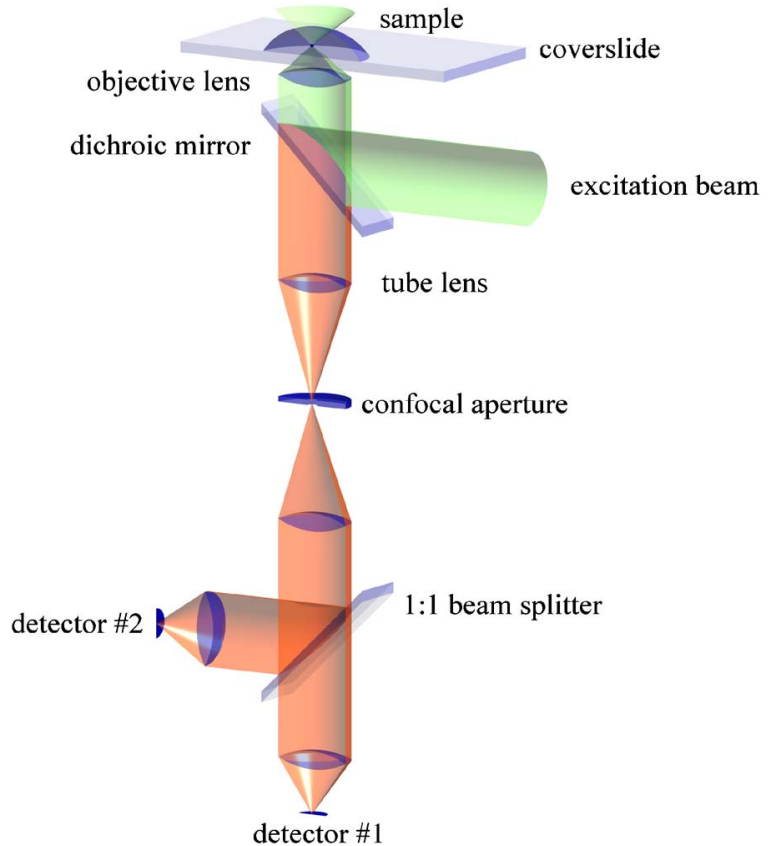

FIG. 2. (Color online) Sketch of the confocal setup used for experimental verification.

over $5 \times 10^{6}$ counts/s are obtained. In T3 mode this total transfer rate is fully available for input channel 1 .

\section{EXPERIMENTAL RESULTS}

The final implementation of the described TCSPC electronics (PicoHarp 300, PicoQuant) was used in various measurement modes to verify basic functionality and timing accuracy. The latter was found to be typically $9 \mathrm{ps} \mathrm{rms}$. Fluorescence lifetimes were routinely obtained in the classic TCSPC histogramming mode. T3 mode was used to obtain fluorescence lifetimes and FCS at the same time. Further results presented here are limited to recording photons from two detectors in T2 mode (Hanbury-Brown/Twiss configuration). The confocal microscope setup used for measurements is shown in Fig. 2. Details were described previously. ${ }^{8} \mathrm{~A}$ pulsed diode laser at $\sim 470 \mathrm{~nm}$ wavelength (LDH-P-C 470 / PDL $800 \mathrm{~B}$, PicoQuant) generating pulses with $\sim 70$ ps pulse width and $40 \mathrm{MHz}$ repetition rate or a $\mathrm{cw} \mathrm{Ar}^{+}$laser (Innova 70-5, Coherent) operating at $488 \mathrm{~nm}$ was used for excitation. The light of the laser was passed through a single-mode optical fiber and subsequently collimated to form a beam with Gaussian beam profile of approximately $2.5 \mathrm{~mm}$ beam waist radius. The beam was spectrally filtered using a narrow-band excitation filter (475AF20, 488NB3, Omega Optical). The beam was then focused through an apochromatic oilimmersion objective $[60 \times, 14$ NA (numerical aperture), Olympus] into the sample solution. Fluorescence was collected by the same objective (epifluorescence setup) and then separated from the excitation light by a dichroic mirror (505DRLP, Omega Optical). After passing an additional bandpass filter (535AF26, Omega Optical), a tube lens with $180 \mathrm{~mm}$ focal length focused it onto a circular pinhole with $100 \mu \mathrm{m}$ diameter. After the pinhole, the light was split into two channels and refocused by achromatic lenses $(60 \mathrm{~mm}$,

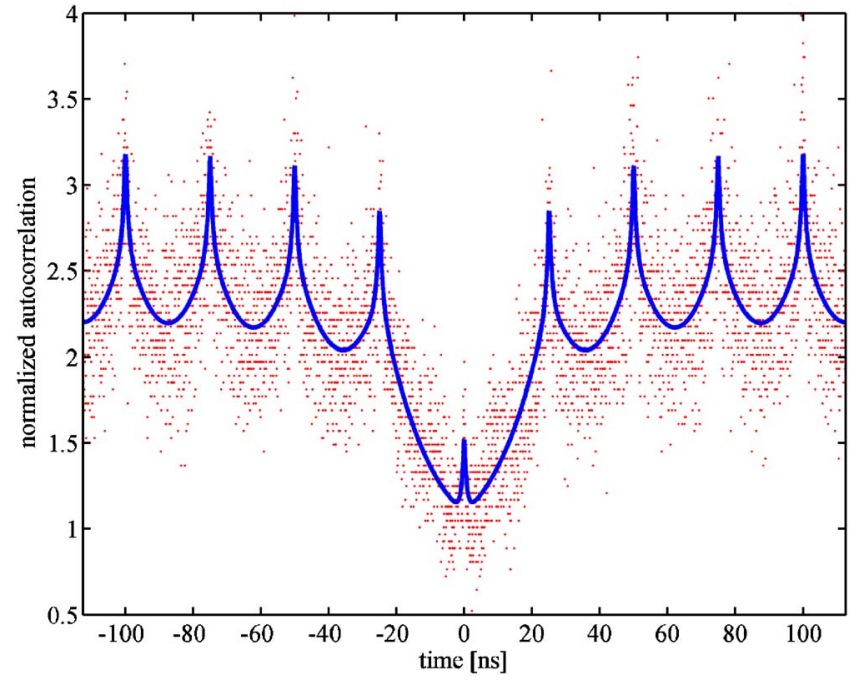

FIG. 3. (Color online) Qdot 525 high resolution fluorescence autocorrelation from T2 mode data at $40 \mathrm{MHz}$ pulsed excitation. Solid line: Model fit according to the photophysics of the sample.

LINOS) onto two single photon avalanche diodes (SPAD) (SPCM AQR-13, Perkin Elmer). The detector output signals were fed to the two timing inputs of the PICOHARP 300. Subsequent software processing of the T2 mode data was done by cross correlating the signals from the two detectors, which eliminates dead time and afterpulsing effects. In terms of the light emitted from the sample this is still an autocorrelation and results are subsequently referred to as the latter.

The first sample consisted of a dilute solution of commercially available quantum dots (Qdot 525, Quantum Dot Corp.) in doubly distilled water. For excitation, the pulsed diode laser was used. The measurement time was $30 \mathrm{~min}$.

Figure 3 shows the autocorrelation function of the photon data on a linear time scale from -100 to $100 \mathrm{~ns}$. Due to the pulsed excitation the fluorescence photons are bunched in distinct time regimes corresponding to the kinetics of the radiative decay. The spacing of $25 \mathrm{~ns}$ of the maxima in the data reflects the repetition rate $(40 \mathrm{MHz})$ of the excitation laser. For only few (less than five) emitters on average in the detection volume one observes a noticeable drop of the correlation for zero lag time due to the impossibility to excite a molecule that is already in the excited state. Using the ratio of the amplitudes of the second order correlation function at zero lag time and at a lag time that corresponds to a multitude of the cycle time of the laser one can derive the number of fluorescent emitters independent from the number of diffusing particles. This ability opens numerous new applications in single molecule spectroscopy. The temporal resolution in the range of a few picoseconds increases the accuracy of the determination of the amplitude of the zero lag time amplitude.

Further analysis was done by fitting the data to a model, which describes the photophysics of the sample. ${ }^{9}$ The model shows excellent agreement with the experimental data. One obtains two lifetimes $\left(\tau_{1}=18 \mathrm{~ns}\right.$ and $\left.\tau_{2}=0.8 \mathrm{~ns}\right)$ for the mono- and biexcitons respectively. Both values agree perfectly with published results. ${ }^{9}$

Figure 4 shows the autocorrelation function of the same 


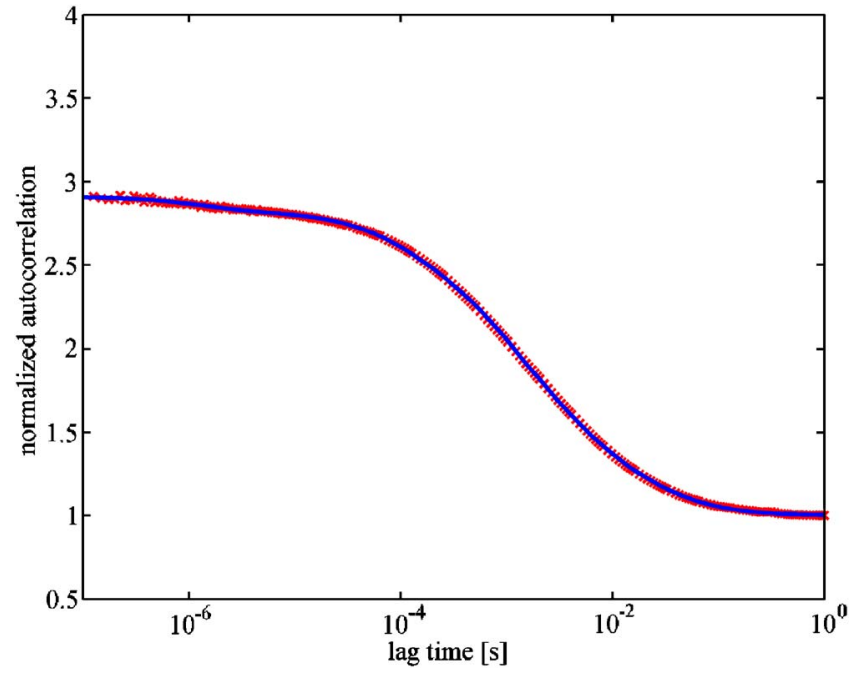

FIG. 4. (Color online) Qdot 525 lower resolution fluorescence autocorrelation from T2 mode data at $40 \mathrm{MHz}$ pulsed excitation. Solid line: FCS model fit.

data in a time range from $250 \mathrm{~ns}$ to $1 \mathrm{~s}$ on a logarithmic time scale. In order to obtain a reasonable fit quality using the standard FCS model of a three-dimensional Gaussian pointspread function ${ }^{10}$ one has to use two additional decay processes that describe the photophysics of the emitter. One obtains a diffusion time of $4 \mathrm{~ms}$. The photophysics leads to a decrease of the average brightness of $\sim 30 \%$, mainly due to a quite slow process $(k=1.9 / \mathrm{ms})$.

In order to demonstrate FCS over the full time range down to the picosecond scale, another experiment was performed with $\mathrm{cw}$ illumination using the $\mathrm{Ar}^{+}$laser. Excitation power was $\sim 20 \mu \mathrm{W}$ in the sample. Fluorescence from a nanomolar aqueous solution of rhodamine 6G (Radiant Dyes, Germany) was recorded for $30 \mathrm{~min}$. Figure 5 shows the correlation result from lag times of 4 ps up to $1 \mathrm{~s}$. Despite the statistical fluctuations at short lag times, the antibunching dip is clearly visible. The data were fitted using an extended FCS model, as described by Felekyan et al. ${ }^{7}$ One observes excellent agreement. The antibunching term reveals a fluorescence lifetime of $\tau \mathrm{fl}=4.1 \mathrm{~ns}$, which corresponds to literature values.

\section{DISCUSSION}

TDC based photon timing on independent channels has some striking advantages over the classical TAC based approach, especially in conjunction with time tagged photon data collection. The time tagging measurement modes allow for performing a wide array of measurement tasks. The concept is without redundancy in the data stream, but also without any loss of information, like e.g., in immediate histogramming. Virtually all algorithms and methods for the analysis of fluorescence dynamics can be implemented.

Intensity traces over time, as traditionally obtained from multichannel scalers (MCSs), are obtained from T2 or T3 data by evaluating only the time tags of the photon records.

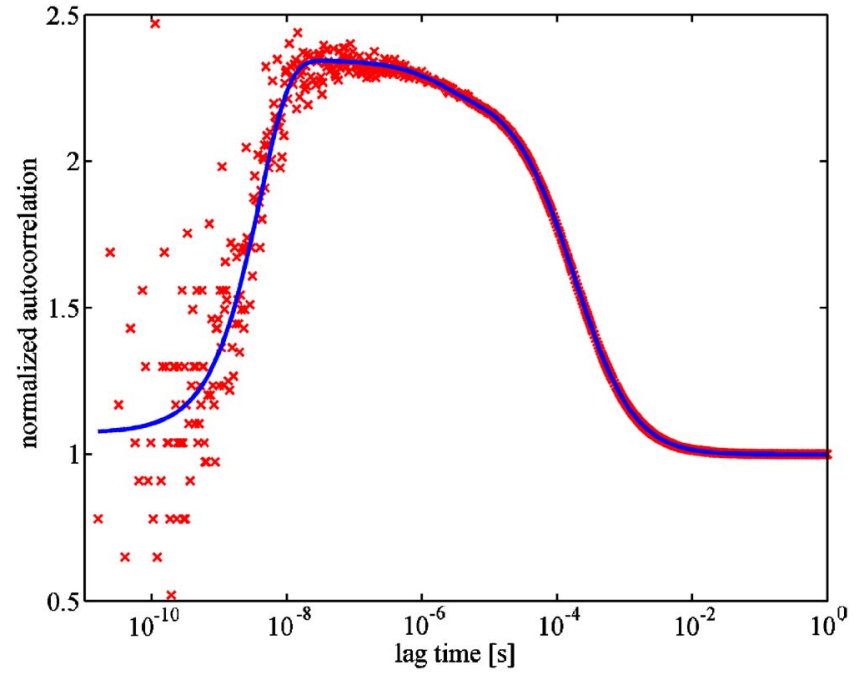

FIG. 5. (Color online) Rhodamine 6G full fluorescence autocorrelation from $\mathrm{T} 2$ mode data at $\mathrm{cw}$ excitation spanning from 4 ps to $1 \mathrm{~s}$. Solid line: Extended FCS model fit.

Sequentially stepping through the arrival times, all photons within the chosen time bins (typically milliseconds) are counted. This gives access to, e.g., single molecule bursts (in flow) or to blinking dynamics. The bursts can be further analyzed by, e.g., histogramming burst height or frequency analysis. Fluorescence lifetimes can be obtained by histogramming the TCSPC (start-stop) times and fitting of the resulting histogram.

The strength of the T3 format is best exploited when both time figures are used together. For instance, one can first evaluate the MCS trace to identify single molecule bursts, and then use the TCSPC times within those bursts to evaluate fluorescence lifetimes. If there are different molecular species with different fluorescence lifetimes, these can be used to distinguish them in real time, e.g., in capillary flow approaches to DNA sequencing or substance screening. Vice versa, one can employ time gating on the TCSPC time before evaluating the intensity trace, i.e., one rejects all photons that do not fall into a time window that is probable to contain fluorescence photons. This reduces noise from background and scattered excitation light.

An advantage of offline analysis of time tagged photon event data is that it can be repeated infinitely with variations in the analysis approach. A good example is FCS. Traditional hardware correlators perform an immediate (real-time) data reduction that does not keep the original data and that prohibits to "slice out" pieces of data generated by sample contaminations (e.g., molecule aggregates and dust particles). Scatter or strong fluorescence from these contaminations will immediately "swamp" the obtained correlation function. Having individual photon records available, one can perform the correlation by software and select the "good" data, or data of interest, as required. On modern computers and with recently developed fast algorithms, ${ }^{11}$ it is possible to perform the correlation even in real time. First experiments with the instrument allowed real-time autocorrelation with 250 tau data points at count rates up to $300 \mathrm{kHz}$ on a Pentium 4 with $2.8 \mathrm{GHz}$ clock. 
Finally, the ultimate strength in T3 mode based FCS analysis is again the combination of the two time figures. As a first useful approach, one can again employ time gating on the TCSPC time to reject scatter and background noise. ${ }^{12}$ Furthermore, it has recently been shown that it is possible to separate different molecular species in a mixture in one single FCS measurement. By filtering the photon events according to their TCSPC time before they enter the correlation procedure, one can obtain separate FCS curves for each species. ${ }^{13}$ A logical step further is cross correlation between the two species. ${ }^{14}$

Yet another application of T3 mode is in fluorescence lifetime imaging (FLIM), which is a powerful extension of fluorescence imaging microscopy. In order to perform FLIM, the spatial origin of the photons must be recorded in addition to the TCSPC data. Conventional FLIM systems use a large array of on-board memory to accommodate the large amount of data generated due to the three-dimensional matrix of pixel coordinates and lifetime histogram channels. Even with modern memory chips, this approach is limiting the recordable image size. To solve the problem we previously extended the TTTR data stream concept to contain markers for synchronization information from, e.g., a piezoscanner. ${ }^{15}$ The same concept, only with more marker signals, was implemented in the present instrument. This makes possible to reconstruct two-dimensional (2D) or threedimensional (3D) images from the stream of T3 records, since the position of the scanner can be determined during data analysis. The data are nearly free of redundancy and can therefore be transferred in real time, even if the scan speed is very fast, like, e.g., in laser scanning microscopes (LSM). ${ }^{16}$ The image size is unlimited both in size and in count depth.

In order to facilitate multiparameter detection such as multiple wavelengths or polarization states, it is a common practice to employ detector multiplexing techniques in TCSPC. ${ }^{17}$ Due to the inherent single photon statistics this multiplexing is actually increasing overall efficiency. For the present instrument such multiplexing devices have been implemented for up to four detectors, supporting histogramming mode as well as T3 mode.

\section{ACKNOWLEDGMENTS}

Part of this work was supported by the Land Berlin "Programm zur Förderung wirtschaftsrelevanter Innovationen" and cofinanced by the EU fund for regional development (EFRE).

${ }^{1}$ D. V. O'Connor and D. Phillips, Time-Correlated Single Photon Counting (Academic, London, 1984).

${ }^{2}$ C. W. Wilkerson, P. M. Goodwin, W. P. Ambrose, J. C. Martin, and R. A. Keller, Appl. Phys. Lett. 62, 2030 (1993).

${ }^{3}$ R. Rigler, J. Widengren, and Ü. Mets, in Fluorescence Correlation Spectroscopy, edited by R. Rigler and E. Elson (Springer, Berlin, 2001).

${ }^{4}$ K. Schätzel, Inst. Phys. Conf. Ser. 77, 175 (1985).

${ }^{5}$ M. Wahl, R. Erdmann, K. Lauritsen, and H. J. Rahn, Proc. SPIE 3259, 173 (1998).

${ }^{6}$ J. Kalisz, Metrologia 41, 1 (2004).

${ }^{7}$ S. Felekyan, R. Kühnemuth, V. Kudryavtsev, C. Sandhagen, W. Becker, and C. A. M. Seidel, Rev. Sci. Instrum. 76, 083104 (2005).

${ }^{8}$ M. Böhmer, F. Pampaloni, M. Wahl, H. J. Rahn, R. Erdmann, and J. Enderlein, Rev. Sci. Instrum. 72, 4145 (2001).

${ }^{9}$ B. Fisher, J. M. Caruge, D. Zehnder, and M. Bawendi, Phys. Rev. Lett. 94, 087403 (2005)

${ }^{10} \mathrm{R}$. Rigler and J. Widengren, in Bioscience, edited by B. Klinge and C. Owman (Lund University Press, Lund, 1990), pp. 180-183.

${ }^{11}$ M. Wahl, I. Gregor, M. Patting, and J. Enderlein, Opt. Express 11, 3583 (2003).

${ }^{12}$ D. C. Lamb, A. Schenk, C. Röcker, C. Scalfi-Happ, and G. U. Nienhaus, Biophys. J. 79, 1129 (2000).

${ }^{13}$ M. Böhmer, M. Wahl, H. J. Rahn, R. Erdmann, and J. Enderlein, Chem. Phys. Lett. 353, 439 (2002).

${ }^{14}$ A. Benda, M. Hof, M. Wahl, M. Patting, R. Erdmann, and P. Kapusta, Rev. Sci. Instrum. 76, 033106 (2005).

${ }^{15}$ F. Koberling, M. Wahl, M. Patting, H. J. Rahn, P. Kapusta, and R. Erdmann, Proc. SPIE 5143, 181 (2003).

${ }^{16}$ U. Ortmann, T. Dertinger, M. Wahl, H. J. Rahn, M. Patting, and R. Erdmann, Proc. SPIE 5325, 179 (2004).

${ }^{17}$ D. J. S. Birch, D. McLoskey, A. Sanderson, K. Suhling, and A. S. Holmes, J. Fluoresc. 04, 91 (1994). 